\title{
Single-path Provisioning with Multi-path Recovery in Flexgrid Optical Networks
}

\author{
Alberto Castro*, Luis Velasco, Marc Ruiz, and Jaume Comellas \\ Advanced Broadband Communications Center (CCABA) \\ Universitat Politècnica de Catalunya (UPC), Barcelona, Spain \\ Email: acastro@ac.upc.edu
}

\begin{abstract}
The new flexgrid technology, in opposition to the fixed grid one traditionally used in wavelength switched optical networks (WSON), allows allocating the spectral bandwidth needed to convey heterogeneous client demand bitrates in a flexible manner so that the optical spectrum can be managed much more efficiently. In this paper we propose a new recovery scheme, called single-path provisioning multi-path recovery (SPP-MPR), specifically designed for flexgrid-based optical networks. It provisions single-paths to serve the bitrate requested by client demands and combines protection and restoration schemes to jointly recover, in part or totally, that bitrate in case of failure. We define the bitrate squeezed recovery optimization (BRASERO) problem to maximize the bitrate which is recovered in case of failure of any single fiber link. A mixed integer linear programming (MILP) formulation is provided. Exhaustive numerical experiments carried out over two network topologies and realistic traffic scenarios show that the efficiency of the proposed SPP-MPR scheme approaches that of restoration mechanisms while providing recovery times as short as protection schemes.
\end{abstract}

Index Terms-Flexgrid optical networks, Single-path Provisioning Multi-path Recovery, Bitrate squeezing.

\section{INTRODUCTION}

$\mathrm{F}$ UTURE flexgrid optical networks featuring flexible and elastic spectrum allocation [1], [2] are attracting high interest from network operators and the research community in general as a result of its higher spectrum efficiency and flexibility with respect to wavelength switched optical networks (WSON) [3], based on the wavelength division multiplexing (WDM) technology. In flexgrid optical networks, the available optical spectrum is divided into frequency slots of fixed spectrum width, e.g. $25 \mathrm{GHz}$ or $12.5 \mathrm{GHz}$. Optical connections (paths) can use a variable number of these slots, depending on the requested bitrate, the modulation format used, and the slot width.

Owing to the huge bitrate associated to each established path, recovery schemes need to be used to guarantee that the associated client connection demands continue being served even in case of failures [4]. As in WSON, recovery can be provided by either protection, where the failed working path is substituted by a pre-assigned backup one, or restoration, which is based on rerouting the working path. Backup paths use resources, i.e. each of the frequency slots in a fiber link, that are dedicated to protect a single working path, or they can be shared to provide protection to multiple working paths. As a consequence, the former scheme is called dedicated path protection (DPP) and the latter shared path protection (SPP).

Although protection schemes reserve resources to guarantee that all the protected paths are recovered in case of any single failure, SPP provides better resource utilization than DPP because spare resources are shared among several working paths. On the one hand, path restoration is the most efficient scheme since resources are only allocated after a failure impacts a working path. On the other hand, recovery times are usually much shorter when protection is used. In the case of DPP, since spare resources are already in use, recovery times are really short, being slightly longer for SPP since spare resources are reserved beforehand and activated in case of failure.

\section{A. Related Work}

Both, protection and restoration schemes have been widely studied in the literature [4]-[14].

Some works have studied multi-path routing considering also the requirements for distributed data-intensive applications. Applied to WSON, authors in [6], [7] propose a mechanism for multiple lightpaths provisioning which satisfies extremely high bandwidth requirements whilst minimizes the amount of traffic affected by single link failures upper-bounding also differential delay. Authors in [8] propose using multi-path provisioning for flexgrid optical networks as a mechanism to increase network performance. The application of multi-path to recovery was introduced by authors in [9] where $K$ disjoint paths are both provisioned and protected using SPP.

The concept of partial protection by provisioning multiple paths was also studied by authors in [10] and extended to mixed-line-rate scenario in [11], [12]. A slightly different partial recovery scheme was introduced in [13] for flexgridbased networks. It consists in provisioning only one path for the full requested bitrate and use restoration in case of failure to find a backup path to recover part of the requested bitrate (bandwidth squeezing); this entails reducing the number of frequency slots that are actually recovered from a failure.

Authors in [14] propose combining protection and restoration aiming at adding extra availability in case of double failures; protection is used to recover from the first failure and restoration to recover from the second one. 


\section{B. Contributions}

In the context of flexgrid optical networks, the contributions of this work are two-fold: $i$ ) we propose using single-path provisioning to avoid differential delay and study protection and restoration mechanisms to recover connections in the event of single-link failures under the bandwidth squeezing assumption. To this end, we define the BitRAte SquEezed Recovery Optimization (BRASERO) problem to maximize the recovered bitrate which is served in case of failure of any single fiber link. The BRASERO problem is modeled as a Mixed Integer Linear Programming (MILP) problem with special constraints for DPP, SPP and restoration, and solved it considering different network scenarios; ii) aiming at providing the best trade-off between the high amount of unrestored bandwidth provided by restoration and the low recovery times inherent to protection, we propose Single-path Provisioning Multi-path Recovery (SPP-MPR), a single-path provisioning scheme together with a multi-path squeezed recovery mechanism which combines protection and restoration schemes. Note that, in contrast to [14], in our proposal protection and restoration paths are simultaneously active and cooperate in recovering the bitrate requested by a single demand. To limit differential delay problems, the number of backup paths is restricted to two; one for protection and another for restoration. The BRASERO MILP formulation gives support for the proposed multi-path recovery scheme.

The remainder of this paper is organized as follows. Section II presents adaptations of protection and restoration schemes to be used in flexgrid optical networks. Besides, the proposed SPP-MPR scheme is also presented. Section III states and models the BRASERO problem as a MILP formulation. The BRASERO problem uses either DPP, SPP, restoration or SPPMPR schemes to recover part of the requested bitrate of each client demand in each failure scenario defined. The proposed MILP model is subsequently applied to different reference network topologies in section IV, where the benefits of using the proposed SPP-MPR scheme are highlighted. Finally, section $\mathrm{V}$ gives some concluding remarks.

\section{RECOVERY SCHEMES IN FLEXGRID NETWORKS}

As stated in the introduction, protection (DPP and SPP) and restoration schemes have been traditionally used in WSON. The efficiency of restoration is, in general, higher than that of SPP, being DPP the scheme with the lowest efficiency. Regarding recovery times, they are usually much shorter in DPP and SPP since spare resources are reserved beforehand.
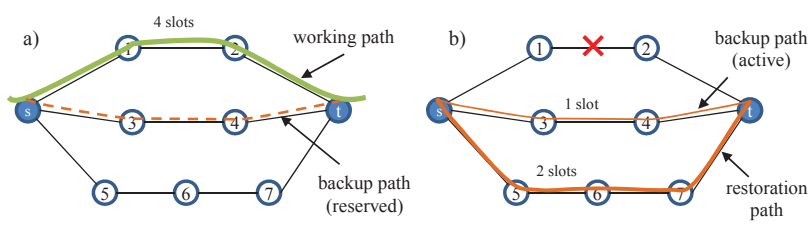

Fig. 1. SPP-MPR in action. In a) $n^{d}=4$ slots are being used along the working path of a demand. Resources for the backup path are reserved. After a failure (b) the backup path is activated and a restoration path has been also established. In total, $n_{\text {recovery }}^{d}=3$ slots $(75 \%)$ are recovered.
On the contrary, restoration lightpaths, including both route and wavelength assignment, need to be found after a failure impacts working lightpaths [4].

In flexgrid optical networks, the bitrate requested by client demands is converted to a number of frequency slots to be allocated. According to [2], the number of slots $n^{d}$ needed to convey the bitrate $b^{d}$ requested by a client demand $d$ can be computed as

$$
n^{d}=\left\lceil\frac{b^{d}}{m \cdot \Delta_{S}}\right\rceil
$$

where $m$ is the spectral efficiency of the modulation format (bit/s/Hz) and $\Delta_{S}$ is the slot width $(\mathrm{GHz})$. Although several modulation formats can, in general, be used as a function of the path length to increase spectrum efficiency, in this paper, and without loss of generality, only the quadrature phase shift keying (QPSK) modulation format is used; thus the parameter $m=2 \mathrm{~b} / \mathrm{s} / \mathrm{Hz}$.

Therefore, Service Level Agreements (SLA) between network operators and clients can now include specific terms so that all or only part of the requested bitrate is restored in case of failure. Let $q^{d}$ be the percentage of bitrate to be assured in case of failure, and hence, the number of slots, $n_{\text {recovery, }}^{d}$, that must be used for recovery demand $d$ can be computed so to $n^{d}{ }_{\text {recovery }} * m^{*} \Delta_{S} \geq q^{d *} b^{d}$.

Authors in [13] propose the bandwidth squeezed restoration scheme to guarantee that a part of the requested bitrate is served in case of failure. Nevertheless, restoration does not fit well for all clients as a consequence of its inherent long recovery times. In the view of that, we propose to extend further bandwidth squeezing to protection schemes such as DPP and SPP so that backup paths guarantee only part of the requested bitrate. We propose the SPP-MPR scheme which combines protection and restoration schemes to provide the best from both schemes, i.e. short recovery times with the highest efficiency. The proposed SPP-MPR scheme consists in ensuring part of the minimum bitrate by protection, complementing the rest by restoration.

For illustrative purposes, Fig. 1 shows how SPP-MPR works. In Fig. 1a one client demand is requesting some amount of bitrate that translates into 4 slots. The working path serves all the requested bitrate and there are some slots reserved along the backup route to guarantee that some minimum bitrate is recovered. After a failure has impacted the working path, the backup path is activated and a restoration
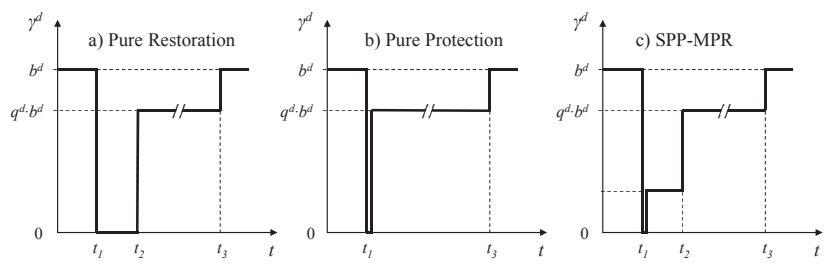

Fig. 2. Evolution of the served bitrate $\left(\gamma^{d}\right)$ for demand $d$ as a function of time when a failure impacts the working path in $t_{l}$. Path restoration (a), Path protection (b), and SPP-MPR (c) schemes are considered. 
path is established in Fig. 1b. Note that both recovery paths jointly guarantee that, at least, $75 \%$ of the requested bitrate is served for that failure.

Fig. 2 presents the evolution of the bitrate actually served $\left(\gamma^{d}\right)$ for a demand $d$ as a function of time when only restoration (Fig. 2a), only protection (Fig. 2b) and a mixed of protection and restoration (Fig. 2c) is used for recovery. In the period $t<t_{1}$ all the requested bitrate is served. In $t=t_{l}$ a failure impacts the working path of demand $d$ and in $t=t_{3}$ the failure is repaired. When only restoration is used for recovery in Fig. 2a, no bitrate is served until the restoration path is established for the demand in $t=t_{2}$. On the opposite, when only protection is used for recovery, the minimum bitrate is served just after the failure is detected in $t=t_{l}$, as shown in Fig. 2b. Since the outage inherent to restoration might not be desirable for some clients and lower efficiency might not be desirable for the network operator, the proposed SPP-MPR scheme serves a part of the minimum bitrate just after the failure is detected in $t=t_{l}$ using protection, while complementing some bitrate using restoration later in $t=t_{2}$, as shown in Fig. 2c. In $t=t_{3}$ the failure is eventually repaired and all the requested bitrate is served again in all the three schemes.

In the next section, the BRASERO problem is formally stated and a MILP-based formulation that uses each of the above-described schemes is proposed.

\section{MATHEMATICAL Formulation}

\section{A. BRASERO problem statement}

The problem can be formally stated as follows:

Given:

- a network topology represented by a graph $\mathrm{G}(N, E)$, where $N$ is the set of optical nodes and $E$ is the set of fiber links connecting two optical nodes,

- a set $S$ of available frequency slots of a given spectral width in each fiber link in $E$,

- a set $D$ of demands to be transported, each requesting a fixed bitrate $b^{d}$, and the minimum bitrate to be guaranteed. For the latter, it can be assumed that $m^{*} \Delta_{S} \mathrm{~Gb} / \mathrm{s}$ must be ensured for each demand.

Output: the routing and spectrum assignment for each $d \in D$, including those scenarios where a failure in a fiber link $e \in E$ impacts the working route of $d$.

Objective: maximize the total recovered bitrate transported in case of failure of any single fiber link $e \in E$ provided that all demands are served in the non-failure scenario.

As previously discussed, the problem can be faced using protection (DPP or SPP), restoration, or a mix of protection and restoration. A MILP-based model which includes the above schemes is presented next.

\section{B. MILP formulation}

The MILP model is based on the link-path formulation [15], where a set of routes are computed beforehand for each demand. It is worth highlighting that the term path is used to describe the set of resources used or reserved to convey a given demand, i.e. a set of contiguous slots along with one route. In addition, the model uses a set of failure scenarios $F$ where one fiber link fails at a time in each of them.

Regarding spectrum allocation, pre-computed channels are used to ensure frequency slot contiguity in the input data, thereby alleviating to some extent the problem complexity [16]. The characteristics of the considered modulation format are also embedded in the input data. To be precise, different optical signal reach values, as a function of both the bitrate and modulation format, are considered.

The following sets and parameters have been defined:

Topology:

$N \quad$ Set of optical nodes, index $n$.

$E \quad$ Set of fiber links, index $e$.

$K \quad$ Set of pre-computed routes, index $k$.

$h_{e}^{k} \quad$ Equal to 1 if route $k$ uses link $e, 0$ otherwise.

len $(k)$ Length of route $k$ in $\mathrm{Km}$.

Spectrum:

$S \quad$ Set of frequency slots available in each link, index $s$.

$\Delta_{S} \quad$ Spectral width $(\mathrm{GHz})$ of each frequency slot.

$m \quad$ Spectral efficiency of the used modulation format ( $m=2 \mathrm{bit} / \mathrm{s} / \mathrm{Hz}$ for QPSK).

$C \quad$ Set of channels, index $c$. Each channel $c$ contains a subset of contiguous slots.

$l_{c s} \quad$ Equal to 1 if channel $c$ uses slot $s, 0$ otherwise.

$n_{c} \quad$ Number of slots of channel $c$.

$R \quad$ Set of bitrate-reach pairs $(\mathrm{Gb} / \mathrm{s}, \mathrm{km})$, index $r$.

len(r) Reach of a path using bitrate-reach pair $r$ in $\mathrm{km}$.

$b(r) \quad$ Maximum bitrate of a path using bitrate-reach pair $r$ in $\mathrm{Gb} / \mathrm{s}$.

Demands:

$D \quad$ Set of optical demands, index $d$.

$b^{d} \quad$ Bitrate requested by demand $d$ in $\mathrm{Gb} / \mathrm{s}$.

$q^{d} \quad$ Percentage of bitrate to be assured in case of failure.

$n^{d} \quad$ Number of frequency slots needed to convey the $n_{\text {recovery }}$ guaranteed bitrate in case of failure.

$d p p \quad$ Binary, equal to 1 if DPP is used, 0 otherwise.

spp Binary, equal to 1 if SPP is used, 0 otherwise.

rest Binary, equal to 1 if restoration is used, 0 otherwise.

$K w(d)$ Set of working routes for demand $d$ (includes reach constraint).

$C(d) \quad$ Set of feasible channels to convey the bitrate requested by demand $d$.

$C p(d) \quad$ Set of feasible channels for demand $d$ so to protect some amount of bitrate in the range $\left[\Delta_{S} \cdot m, \max \right.$ $\left.\left(\Delta_{S} \cdot m, b^{d}\right)\right]$.

$\operatorname{Cr}(d)$ Set of feasible channels for demand $d$ so to restore some amount of bitrate in the range $\left[0, b^{d}\right]$. 
Failures and others:

$F \quad$ Set of failure scenarios, $\operatorname{index} f$.

$K p^{k}(d)$ Subset of backup routes for demand $d$ disjoint to working route $k$.

$K p(d)$ Set of backup routes for demand $d$.

$a_{e f} \quad$ Equal to 1 if fiber link $e$ is available under failure scenario $f$.

$a_{f}^{k} \quad$ Equal to 1 if route $k$ is available under failure scenario $f\left(a_{f}^{k}=\prod_{\{e: h e=1\}}^{k} a_{e f}\right)$.

$u_{f} \quad$ Probability of failure scenario $f\left(u_{f} \in[0,1]\right)$.

The decision variables are:

$\omega^{d k}{ }_{c}$ Binary. Equal to 1 if demand $d$ uses route $k$ and channel $c$ for working, 0 otherwise.

$\kappa^{d k}{ }_{c} \quad$ Binary. Equal to 1 if demand $d$ uses route $k$ and channel $c$ for protection, 0 otherwise.

$\kappa_{c f}^{d k}$ Binary. Equal to 1 if demand $d$ uses route $k$ and channel $c$ for restoration in failure scenario $f, 0$ otherwise.

$\tau^{d} \quad$ Positive real. Protected bitrate for demand $d$.

$\tau_{f}^{d} \quad$ Positive real. Protected bitrate for demand $d$ in failure scenario $f$.

$\varphi_{f}^{d} \quad$ Positive real. Restored bitrate for demand $d$ in failure scenario $f$.

$\gamma_{f}^{d} \quad$ Positive real. Recovered (protected + restored) bitrate for demand $d$ in failure scenario $f$.

$v^{d}{ }_{r} \quad$ Binary. Equal to 1 if demand $d$ uses bitrate-reach pair $r, 0$ otherwise.

$v_{r f}^{d} \quad$ Binary. Equal to 1 if demand $d$ uses bitrate-reach pair $r$ in failure scenario $f, 0$ otherwise.

$\delta_{e s} \quad$ Binary. Equal to 1 if slot $s$ in fiber link $e$ is used by any backup route, 0 otherwise.

$\sigma^{d d^{\prime}} \quad$ Binary. Equal to 1 if working routes of two demands $d$ and $d$ ' share at least one fiber link, 0 otherwise.

The MILP formulation for the BRASERO problem is as follows:

(BRASERO) Max $\Phi=\frac{1}{\sum_{f \in F} u_{f}} \cdot \sum_{f \in F} \sum_{d \in D} u_{f} \cdot \gamma_{f}^{d}$

s. t.

Common constraints:

$\sum_{k \in K w(d)} \sum_{c \in C(d)} \omega_{c}^{d k}=1 \quad \forall d \in D$

$\gamma_{f}^{d} \leq b^{d} \quad \forall d \in D, f \in F$

$\gamma_{f}^{d} \geq n_{\text {recovery }}^{d} \cdot \Delta_{S} \cdot m \quad \forall d \in D, f \in F$

$\gamma_{f}^{d} \leq \tau_{f}^{d}+\varphi_{f}^{d} \quad \forall d \in D, f \in F$

Constraints for path protection:

$\sum_{d \in D} \sum_{k \in K p(d)} \sum_{c \in C p(d)} \kappa_{c}^{d k} \leq(s p p+d p p) \cdot|D|$ $\sum_{k^{\prime} \in K p^{k}(d)} \sum_{c \in C p(d)} \kappa_{c}^{d k^{\prime}} \leq \sum_{c \in C(d)} \omega_{c}^{d k} \quad \forall d \in D, k \in K w(d)$

$\tau^{d} \leq \sum_{k \in K p(d)} \sum_{c \in C p(d)} n_{c} \cdot \Delta_{S} \cdot m \cdot \kappa_{c}^{d k} \quad \forall d \in D$

$\tau^{d} \leq \sum_{r \in R} b(r) \cdot v_{r}^{d} \quad \forall d \in D$

$\tau^{d} \geq \Delta_{S} \cdot m \cdot(s p p+d p p) \quad \forall d \in D$

$\sum_{k \in K p(d)} \sum_{c \in C p(d)} \operatorname{len}(k) \cdot \kappa_{c}^{d k} \leq \sum_{r \in R} \operatorname{len}(r) \cdot v_{r}^{d} \quad \forall d \in D$

$\sum_{r \in R} v_{r}^{d} \leq 1 \quad \forall d \in D$

$s p p \cdot \sum_{d \in D} \sum_{k \in K p(d)} \sum_{c \in C p(d)} h_{e}^{k} \cdot l_{c s} \cdot \kappa_{c}^{d k} \leq \delta_{e s} \cdot|D| \quad \forall e \in E, s \in S$

$\operatorname{spp} \cdot \sum_{k \in K w(d)} \sum_{c \in C(d)} h_{e}^{k} \cdot \omega_{c}^{d k}+\sum_{k \in K w\left(d^{\prime}\right)} \sum_{c \in C\left(d^{\prime}\right)} h_{e}^{k} \cdot \omega_{c}^{d^{\prime} k} \leq 1+\sigma^{d d^{\prime}}$

$\forall d, d^{\prime} \in D, d \neq d^{\prime}, e \in E$

$s p p \cdot \sum_{k \in K p(d)} \sum_{c \in C p(d)} h_{e}^{k} \cdot l_{c s} \cdot \boldsymbol{\kappa}_{c}^{d k}+\sum_{k \in K p\left(d^{\prime}\right)} \sum_{c \in C p\left(d^{\prime}\right)} h_{e}^{k} \cdot l_{c s} \cdot \boldsymbol{\kappa}_{c}^{d^{\prime} k}$

$\leq 2-\sigma^{d d^{\prime}} \quad \forall d, d^{\prime} \in D, d \neq d^{\prime}, e \in E, s \in S$

$d p p \cdot \sum_{d \in D} \sum_{k \in K p(d)} \sum_{c \in C p(d)} h_{e}^{k} \cdot l_{c s} \cdot \kappa_{c}^{d k} \leq \delta_{e s} \quad \forall e \in E, s \in S$

$\sum_{d \in D} \sum_{k \in K w(d)} \sum_{c \in C(d)} h_{e}^{k} \cdot l_{c s} \cdot \omega_{c}^{d k} \leq\left(1-\delta_{e s}\right) \quad \forall e \in E, s \in S$

$\tau_{f}^{d} \leq a_{f}^{k} \cdot b^{d}+\left(1-a_{f}^{k}\right) \cdot \tau^{d}+M \cdot\left(1-\omega_{c}^{d k}\right)$

$\forall d \in D, k \in K w(d), c \in C(d), f \in F$

Constraints for path restoration:

$\sum_{d \in D \in K p(d)} \sum_{c \in C r(d)} \sum_{f \in F} \kappa_{c f}^{d k} \leq$ rest $\cdot|D| \cdot|F|$

$\sum_{k \in K p(d)} \sum_{c \in C r(d)} \kappa_{c f}^{d k} \leq \sum_{k \in K w(d)} \sum_{c \in C(d)}\left(1-a_{f}^{k}\right) \cdot \omega_{c}^{d k} \forall d \in D, f \in F$

$\varphi_{f}^{d} \leq \sum_{k \in K p(d)} \sum_{c \in C r(d)} n_{c} \cdot \Delta_{S} \cdot m \cdot \kappa_{c f}^{d k}+\sum_{k \in K w(d)} \sum_{c \in C(d)} a_{f}^{k} \cdot b^{d} \cdot \omega_{c}^{d k}$

$\forall d \in D, f \in F$

$\varphi_{f}^{d} \leq \sum_{r \in R} b(r) \cdot v_{r f}^{d} \quad \forall d \in D, f \in F$

$\sum_{k \in K p(d)} \sum_{c \in C r(d)} l e n(k) \cdot \kappa_{c f}^{d k} \leq \sum_{r \in R} l e n(r) \cdot v_{r f}^{d} \quad \forall d \in D, f \in F$

$\sum_{r \in R} v_{r f}^{d} \leq 1 \quad \forall d \in D, f \in F$

$\sum_{d \in D \in \in K w(d)} \sum_{c \in C(d)} a_{f}^{k} \cdot h_{e}^{k} \cdot l_{c s} \cdot \omega_{c}^{d k}+\sum_{d \in D} \sum_{k \in K p(d)} \sum_{c \in C r(d)} h_{e}^{k} \cdot l_{c s} \cdot \kappa_{c f}^{d k} \leq$
$\quad \leq a_{e f} \cdot\left(1-\delta_{e s}\right) \quad \forall e \in E, s \in S, f \in F$

The objective function (2) maximizes the total bitrate recovered. The bitrate recovered in each failure scenario is weighted by the probability of that scenario.

Constraint (3) ensures that the bitrate requested by each demand is served in the non-failure scenario, i.e. one working path (route and channel) is assigned to each demand. Constraints (4)-(5) limit the recovered bitrate of each demand 
in each failure scenario to some amount of bitrate in between the requested and the guaranteed ones. Constraint (6) computes the bitrate conveyed for each demand in each failure scenario, which can be simultaneously protected and restored when a failure impacts the working path for that demand.

Constraints (7) to (13) deal with the protection of demands, i.e. guaranteeing a minimum protected bandwidth per demand subject to path protection constraints. Constraint (7) makes sure that protection paths are allowed if and only if protection is permitted. Constraint (8) selects, at most, one backup route, disjoint to the working one, for each demand. In addition, one channel is chosen. Constraints (9)-(11) compute the protected bitrate for each demand, which is limited by the size of the selected channel (9), and the maximum bitrate allowed by the bitrate-reach pair selected (10), ensuring that, at least, one slot is restored by protection (11). Constraint (12) guarantees that the length of the backup route chosen does not exceed the reach selected. Constraint (13) assures that one and only one bitrate-reach pair is selected for each demand.

Constraints (14)-(18) deal with the use of slots. When SPP is selected, constraint (14) allows slots to be shared by a number of backup paths, whereas constraints (15)-(16) manage slot sharing; constraint (15) checks whether the working routes of two demands share at least one fiber link and constraint (16) prevents the backup paths of two demands whose working routes share some fiber link from sharing any slot in common links. In contrast, when DPP is selected, constraint (17) makes sure that a slot is used by no more than one backup path. Constraint (18) guarantees that each slot is used at most by one working path when it is not used for protection.

Constraint (19) together with (4)-(6) computes the bitrate conveyed for each demand in each failure scenario, which equals $\tau^{d}$ when a failure impacts the working path for that demand and $b^{d}$ on the contrary.

Constraints (20) to (26) deal with restoration. Constraint (20) controls whether restoration is allowed. Constraint (21) allows finding a restoration path for each demand and failure scenario if and only if the failure affects the working path of the demand. Constraints (22)-(23) compute the restored bitrate for each demand, which is limited when the failure impacts the demand by the size of the selected channel (22) and the maximum bitrate allowed by the bitrate-reach pair selected (23). Constraint (24) ensures that the length of each restoration route chosen do not exceed the reach selected. Constraint (25) guarantees that one and only one bitrate-reach pair is selected for each demand and failure scenario. Constraint (26) deals with the use of slots in case of failure; it guarantees that each slot is used at most by either one working path or one restoration path, provided that it is not already in use for protection.

\section{Complexity Analysis}

The BRASERO problem is NP-hard since simpler network routing problems have been proved to be NP-hard (e.g., [17]). As to the BRASERO problem size, the number of variables

\begin{tabular}{|c|c|c|}
\multicolumn{1}{c|}{} & TABLE I Size OF THE BRASERo Problem \\
\cline { 2 - 3 } \multicolumn{1}{c|}{} & Constraints & Variables \\
\hline \multirow{2}{*}{ Common } & $\mathrm{O}(|D| \cdot|F|)$ & $\mathrm{O}(|D| \cdot|K| \cdot|C|+|D| \cdot|F|)$ \\
& $\left(4 \cdot 10^{3}\right)$ & $\left(10^{7}\right)$ \\
\hline \multirow{2}{*}{ DPP } & $\mathrm{O}(|D| \cdot|K| \cdot|C| \cdot|F|+$ & $\mathrm{O}(|D| \cdot|K| \cdot|C|+$ \\
& $|E| \cdot|S|)$ & $|D| \cdot|R|+|E| \cdot|S|)$ \\
& $\left(2 \cdot 10^{8}\right)$ & $\left(10^{7}\right)$ \\
\hline \multirow{2}{*}{ SPP } & $\mathrm{O}(|D| \cdot|K| \cdot|C| \cdot|F|+$ & $\mathrm{O}\left(|D|^{2}+|D| \cdot|K| \cdot|C|+\right.$ \\
& $\left.|D|^{2} \cdot|E| \cdot|S|\right)$ & $|D| \cdot|R|+|E| \cdot|S|)$ \\
Path & $\left(3 \cdot 10^{8}\right)$ & $\left(10^{7}\right)$ \\
\hline Restoration & $\mathrm{O}(|D| \cdot|F|+|E| \cdot|S| \cdot|F|)$ & $\mathrm{O}(|D| \cdot|K| \cdot|C| \cdot|F|+$ \\
& $\left(5 \cdot 10^{4}\right)$ & $|D| \cdot|R| \cdot|F|)+|E| \cdot|S|)$ \\
& & $\left(2 \cdot 10^{8}\right)$ \\
\hline
\end{tabular}

and constraints are depicted in Table I for each constraint group defined in the model above; the complete size of the problem is obtained adding the size of each group of constraints. Additionally, an estimation of the BRASERO problem size is calculated for the DT network and the scenario presented in Section IV.

Although the MILP can be solved for small instances, its exact solving becomes impractical for realistic backbone networks (under appreciable load) such as those described in Section IV, even using commercial solvers such as CPLEX [18]. Thus, aiming at providing near-optimal solutions within reasonable computational effort, a Biased Random-Key Genetic Algorithm (BRKGA) -based meta-heuristic [19] algorithm is used to solve the BRASERO problem. Although, due to lack of space the used algorithm is not described, it is worth highlighting that the heuristic was validated, using small instances, against the MILP formulation; in all the small instances checked, the heuristic provided the optimal solution, i.e. the same solution than the one obtained from solving the MILP model with CPLEX. Regarding computation times, few minutes were enough for the heuristic, in contrast to several hours needed to obtain the same solution using CPLEX. Thus, we used the heuristic algorithm to solve the larger instances presented in the next Section.

\section{ILLUSTRATIVE NUMERICAL RESUltS}

In this Section, we first present the network scenarios that we consider in order to carry out our experiments. Secondly, we show the results of solving the BRASERO problem considering a set of realistic traffic instances.

\section{A. Network Scenario}

In order to conduct all the experiments, we consider the two optical network topologies shown in Fig. 3. These are the 10node 16-link Spanish Telefónica (TEL) topology and the 10node 18-link Deutsche Telekom (DT) topology. In addition, two frequency slot widths are considered, namely $25 \mathrm{GHz}$ and 12.5 GHz. In all the experiments the entire spectrum is set to 1.6 THz, i.e. $6425 \mathrm{GHz}$ slots or $12812.5 \mathrm{GHz}$ slots.

As for the traffic, in line with [20], we make use of two traffic profiles (TP) where demand bitrates are 10, 40, 100, or $400 \mathrm{~Gb} / \mathrm{s}$. The TPs selected range from a scenario with a high number of 10 and $40 \mathrm{~Gb} / \mathrm{s}$ connection demands and $52 \mathrm{~Gb} / \mathrm{s}$ on average (TP-1) to a scenario with fewer demands but with a 
higher bit-rate $(80 \mathrm{~Gb} / \mathrm{s}$ on average in TP-2). Indeed, these TPs are a realistic representation of the expected evolution of bandwidth necessities for the years to come. It is worth noting, however, that the average amount of $\mathrm{Tb} / \mathrm{s}$ offered to the network is equal for all TPs. Finally, we assume a $q^{d}$ value so that $n_{\text {recovery }}^{d}=1$ for all the demands.

\section{B. Performance evaluation of protection schemes}

Fig. 4 plots the percentage of unrecovered bitrate against the total network load $(\mathrm{Tb} / \mathrm{s})$. Each point in the plots represents the average value from five independent instances, where the demand matrix of each instance consists in uniformly distributed origin-destination pairs which requested bitrate follows the above-defined TPs. Four graphs per network scenario are represented, one per each TP and frequency slot width pair. Each graph plots the obtained performance of every studied recovery schemes. It is worth noting that when the network load increases, the amount of unrecovered also increases until the problem becomes infeasibility as a result of $i$ ) one or more demands could not be provisioned or $i$ ) the minimum bitrate to be recovered could not be guaranteed for one or more demands in one or more failure scenarios. Hence, feasible solutions serve all the traffic and guarantee the minimum bitrate in all the failure scenarios.

As expected, the restoration approach (PR) clearly supports more traffic load than the protection ones in all the network scenarios studied. However SPP outperforms DPP; while the traffic increment of restoration with respect to DPP is meaningful, $33 \%$ on average, that ratio for SPP is only $9 \%$. This is as a result of a better spare resources usage of SPP. Obviously SPP-MPR-based schemes behave the same as their pure protection counterpart schemes, since guaranteed bitrate

is recovered also by protection (DPP or SPP).

Interestingly, the maximum amount of served traffic is higher when the $12.5 \mathrm{GHz}$ slot width is implemented when compared to that of the $25 \mathrm{GHz}$. The rationale behind that is the higher spectral efficiency of the narrower slot width, as concluded in [20].

Comparing mixed schemes against pure protection schemes we observe, for the same traffic load, a clear gain in the amount of unrecovered bitrate when restoration is added in SPP-MPR-based schemes. Note that, due to the higher efficiency of restoration, more bitrate can be recovered. Table II summarizes the gains obtained by SPP-MPR-based with respect to pure protection schemes for the highest load supported traffic load, for each network topology and TP considered. Gains as high as $54 \%$ are observed clearly demonstrating the goodness of the proposed scheme.
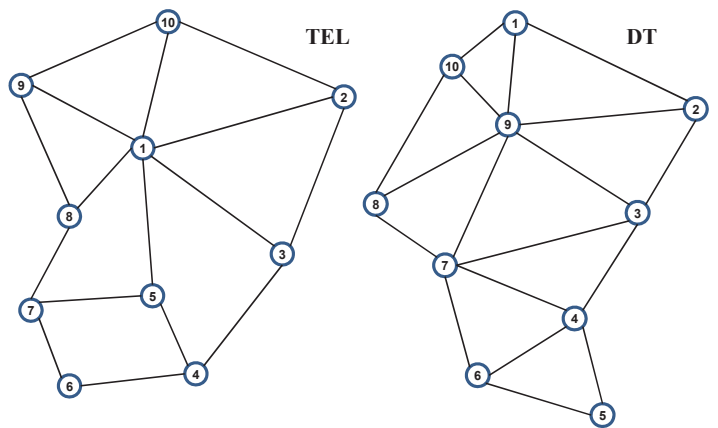

Fig. 3. Sample optical network topologies used in this paper: 10-node Spanish Telefónica (left), 10-node Deutsche Telecom (right).
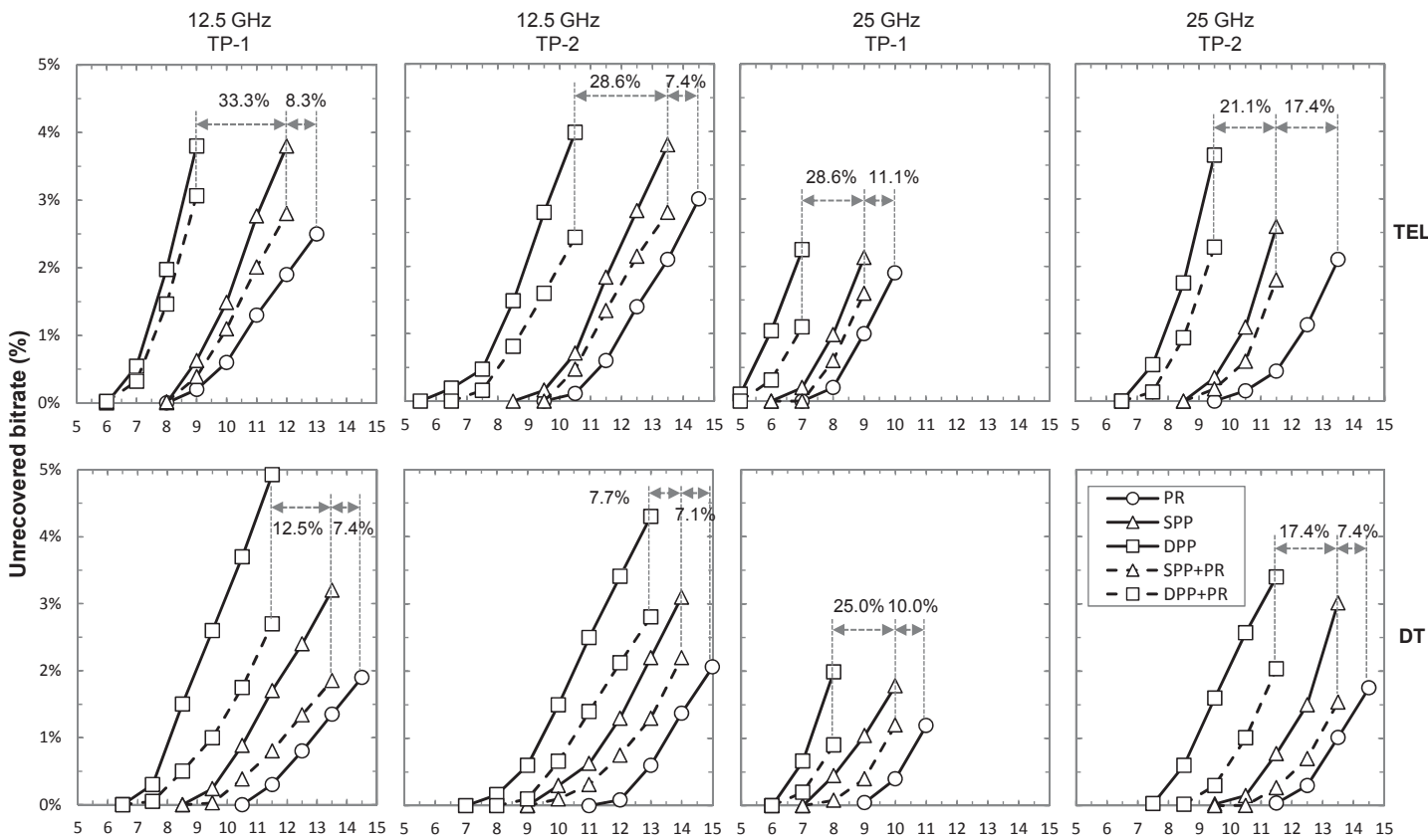

Total Load (Tb/s)

Fig. 4. Unrecovered bitrate (\%) vs. the total network load (Tb/s) for the different TPs, slots widths, and network topologies. 
TABLE II UNRECOVERED BITRATE GAIN AT HigHEST LOAD WHEN SPPMPR-BASED SCHEMES ARE USED

\begin{tabular}{|c|c|c|c|c|c|}
\cline { 3 - 6 } \multicolumn{2}{c|}{} & \multicolumn{2}{c|}{ TEL } & \multicolumn{2}{c|}{ DT } \\
\hline \multirow{2}{*}{ TP } & $\begin{array}{c}\text { Slot Width } \\
\text { (GHz) }\end{array}$ & $\begin{array}{c}\text { DPP }+ \\
\text { PR }\end{array}$ & $\begin{array}{c}\text { SPP }+ \\
\text { PR }\end{array}$ & $\begin{array}{c}\text { DPP + } \\
\text { PR }\end{array}$ & $\begin{array}{c}\text { SPP + } \\
\text { PR }\end{array}$ \\
\hline \multirow{2}{*}{$\mathbf{1}$} & $\mathbf{2 5}$ & $51.0 \%$ & $24.8 \%$ & $54.7 \%$ & $32.7 \%$ \\
\cline { 2 - 6 } & $\mathbf{1 2 . 5}$ & $19.4 \%$ & $26.3 \%$ & $45.2 \%$ & $42.1 \%$ \\
\hline \multirow{2}{*}{2} & $\mathbf{2 5}$ & $37.4 \%$ & $30.5 \%$ & $40.1 \%$ & $49.0 \%$ \\
\cline { 2 - 6 } & $\mathbf{1 2 . 5}$ & $39.1 \%$ & $26.3 \%$ & $34.7 \%$ & $29.0 \%$ \\
\hline
\end{tabular}

\section{CONCLUding REMARKS}

In this work a novel recovery scheme named single-path provisioning with multi-path recovery (SPP-MPR) specifically designed for flexgrid optical networks has been proposed. SPP-MPR can be applied to recover the requested bitrate of client connection demands in the event of single-link failures. It is important to highlight that partial recovery, i.e. only some proportion of the total requested bitrate is recovered from a failure, was assumed to be included in the SLA contracts between the network operator and the clients.

To compare the performance of SPP-MPR against existing single-path pure recovery schemes, such as DPP, SPP, and path restoration, the BRASERO problem was stated and modeled as a MILP problem.

The performance of all recovery schemes was analyzed through intensive numerical experiments. For the sake of a comprehensive study, two different network topologies, slot widths, and traffic profiles were considered.

The results clearly showed the benefits of the proposed SPP-MPR recovery scheme when compared to pure protection ones. In addition, recall that recovery times are shorter when SPP-MPR is used compared to pure path restoration. Therefore, SPP-MPR provides a good trade-off between efficiency and recovery times.

\section{ACKNOWLEDGMENTS}

The research leading to these results has received funding from the European Community's Seventh Framework Programme FP7/2007-2013 under grant agreement $n^{\circ} 247674$ STRONGEST project. Moreover, it was supported by the Spanish science ministry through the TEC2011-27310 ELASTIC project.

\section{REFERENCES}

[1] M. Jinno, H. Takara, B. Kozicki, Y. Tsukishima, Y. Sone and S. Matsuoka, "Spectrum-efficient and scalable elastic optical path network: architecture, benefits, and enabling technologies," IEEE Commun Mag., vol. 47, pp. 66-73, 2009

[2] M. Jinno, B. Kozicki, H. Takara, A. Watanabe, Y. Sone, T. Tanaka and A. Hirano, "Distance-adaptive spectrum resource allocation in spectrumsliced elastic optical path network," IEEE Commun. Mag., vol. 48, pp. 138-145, 2010.

[3] X. Wang, Q. Zhang, I. Kim, P. Palacharla, and M. Sekiya, "Blocking Performance in Dynamic Flexible Grid Optical Networks - What is the Ideal Spectrum Granularity?” in Proc. ECOC, 2011.
[4] W. Grover, "Mesh-based Survivable Transport Networks: Options and Strategies for Optical, MPLS, SONET and ATM Networking," Prentice Hall, 2003.

[5] C. Ou, J. Zhang, H. Zang, L. Sahasrabuddhe, and B. Mukherjee, "New and Improved Approaches for Shared-Path Protection in WDM Mesh Networks," IEEE/OSA J. Lightwave Technol., vol. 22, pp. 1223-1232, 2004.

[6] X. Chen, M. Chamania, A. Jukan, A. Drummond, N. Fonseca, "On the Benefits of Multipath Routing for Distributed Data-intensive Applications with High Bandwidth Requirements and Multidomain Reach," in Proc. CNSR, 2009.

[7] X. Chen, M. Chamania, A. Jukan, A. Drummond, N. Fonseca, "A Multipath Routing Mechanism in Optical Networks with Extremely High Bandwidth Requests," in Proc. IEEE GLOBECOM, 2009.

[8] A. Pages, J. Perello, S. Spadaro, "Lightpath Fragmentation for Efficient Spectrum Utilization in Dynamic Elastic Optical Networks," in Proc. ONDM, 2012.

[9] S. Huang, C. Martel, B. Mukherjee, "Survivable Multipath Provisioning With Differential Delay Constraint in Telecom Mesh Networks," IEEE/ACM Transactions on Networking, vol. 19, pp. 657-669, 2011.

[10] A. Das, C. Martel, B. Mukherjee, "A Partial-Protection Approach Using Multipath Provisioning," in Proc. IEEE ICC, 2009.

[11] C. Vadrevu, R. Wang, B. Mukherjee, "Degraded Services in MixedLine-Rate Networks Using Multipath Routing," in Proc. ANTS, 2011.

[12] C. Vadrevu, R. Wang, M. Tornatore, C. Martel, B. Mukherjee, "Survivable Provisioning in Mixed-Line-Rate Networks Using Multipath Routing," in Proc. OSA OFC, 2012.

[13] Y. Sone, A. Watanabe, W. Imajuku, Y. Tsukishima, B. Kozicki, H. Takara, and M. Jinno, "Bandwidth Squeezed Restoration in SpectrumSliced Elastic Optical Path Networks (SLICE)," IEEE/OSA J. of Opt. Commun. and Netw., vol. 3, pp. 223-233, 2011.

[14] M. Clouqueur, W. Grover, "Mesh-restorable networks with enhanced dual-failure restorability properties," Springer Photonic Network Communications, vol. 9, pp. 7-18, 2005.

[15] M. Pióro and D. Medhi, Routing, Flow, and Capacity Design in Communication and Computer Networks, Elsevier, 2004.

[16] L. Velasco, M. Klinkowski, M. Ruiz, and J. Comellas, "Modeling the Routing and Spectrum Allocation Problem for Flexgrid Optical Networks," Springer Photonic Network Communications, DOI: 10.1007/s11107-012-0378-7, 2012.

[17] R. Ramaswami, K.N. Sivarajan, "Routing and wavelength assignment in all-optical networks," IEEE/ACM Transactions on Networking, vol. 3, pp. 489-500, 1995.

[18] CPLEX, http://www-01.ibm.com/ software/ integration/ optimization/ cplex-optimizer/.

[19] J. Gonçalves and M. Resende, "Biased random-key genetic algorithms for combinatorial optimization," J. Heuristics, vol. 17, pp. 487-525, 2011 .

[20] A. Castro, L. Velasco, M. Ruiz, M. Klinkowski, J. P. FernándezPalacios, D. Careglio, "Dynamic Routing and Spectrum (Re)Allocation in Future Flexgrid Optical Networks," Elsevier Computer Networks, DOI 10.1016/j.comnet.2012.05.001, 2012. 